\title{
Comparison of the Effects of Curcumin, Tramadol and Surgical Treatments on Neuropathic Pain Induced by Chronic Constriction Injury in Rats
}

\author{
Dilek CEYHAN ${ }^{1}$, Atacan Emre KOCMAN², Engin YILDIRIM ${ }^{3}$, Orhan OZATIK ${ }^{4}$, Sule AYDIN³ ${ }^{3}$ Aydan KOSE² \\ ${ }^{1}$ Osmangazi University, Medical Faculty, Department of Anesthesiology and Reanimation, Eskisehir, Turkey \\ ${ }^{2}$ Osmangazi University, Medical Faculty, Department of Plastic and Reconstructive Surgery, Eskisehir, Turkey \\ ${ }^{3}$ Osmangazi University, Medical Faculty, Department of Pharmacology, Eskisehir, Turkey \\ ${ }^{4}$ Osmangazi University, Medical Faculty, Department of Obstetrics and Gynecology, Reproductive Medicine Unit, Eskisehir, Turkey
}

\section{ABSTRACT}

AIM: Nerve entrapment syndromes are the most common causes of neuropathic pain. Surgical decompression is the preferred method of treatment. The aim of this study was to compare the efficacy of curcumin, tramadol and chronic constriction release treatment (CCR), individually or together, in a rat model of sciatic nerve injury.

MATERIAL and METHODS: Eighty male rats were divided into eight study groups. Group 1 was the sham group. Group 2 was the control group with established chronic constriction injury $(\mathrm{CCl})$. $\mathrm{CCl}$ was also established in Groups 3-8. Group 3 underwent chronic constriction release (CCR). Groups 4 and 5 received curcumin and tramadol. Groups 6 and 7 also received curcumin (100 $\mathrm{mg} / \mathrm{kg}$ daily, oral) and tramadol (10 mg/kg daily, intraperitoneal, 14 days) after CCR, respectively. Combined curcumin-tramadol treatment was applied to Group 8. Behavioral tests (thermal hyperalgesia, dynamic plantar, cold plate test) were performed on days $0,3,7,13,17$, and 21 . Tissue tumor necrosis factor- $a$ (TNF-a) and interleukin-10 (IL-10) levels were analyzed in the nerve and dorsal root ganglion (DRG) samples on day 21. Histopathological examination was performed on the nervous tissue and DRG.

RESULTS: Tramadol-CCR and tramadol-curcumin significantly attenuated mechanical allodynia and thermal hyperalgesia. In the CCl-CCR-tramadol treatment group, TNF-a levels were significantly lower in the sciatic nerve tissue, and DRG and IL-10 levels were significantly higher in the sciatic nerve tissue.

CONCLUSION: CCI-CCR-tramadol treatment is highly effective in the symptomatic treatment of neuropathic pain. CCR-curcumin is associated with less degeneration and high levels of regeneration in the nerve tissue.

KEYWORDS: Curcumin, Nerve injury, Neuropathic pain, Surgery, Tramadol

\section{INTRODUCTION}

$\mathrm{N}$ europathic pain is defined as pain from lesions in both the central and peripheral nervous systems. Nerve entrapment syndromes such as carpal tunnel syndrome, spinal root compression, and trigeminal neuralgia due to vascular compression are the most common causes of neuropathic pain. This pain develops secondary to the chronic compression of peripheral nerves. Increased chronic pressure on a nerve alters blood flow and leads to epineurial ischemia. This can cause inflammation, fibrosis, demyelination, and ultimately axonal loss (5). Such cells secrete pro- and antiinflammatory cytokines, such as tumor necrosis factor-a (TNF-a), interleukin-6 (IL-6), and IL-10, and these substances play an important role on the initiation and development of chronic pain states (20). In addition, TNF-a also induces axonal damage (19). Consequently, spinal glial activation occurs, causing neuropathic pain progression (10). 
In clinical practice, surgical decompression is the preferred method of treatment for nerve entrapment syndromes. Prior to surgical treatment or in situations when surgical treatment cannot be performed, different types of drugs such as local steroids, anticonvulsive drugs, and antidepressants may be used for treatment (5).

Curcumin (diferuloylmethane) is a yellow-pigmented fraction of Curcuma longa. The anti-oxidant, anti-inflammatory, and analgesic effects of curcumin have been reported in many studies $(3,14,15,18)$. It has shown efficacy in the treatment of neuropathic pain (6). Moreover, curcumin has prevented hyperalgesia by altering adrenergic and serotonergic descending inhibition in the brainstem $(2,11)$. Tramadol hydrochloride is a synthetic opioid from the aminocyclohexanol group and is another drug used in neuropathic pain therapy (13). Tramadol acts on the $\mu$-opioid receptor and suppresses spinal dorsal horn neuronal activation (7).

Chronic constriction injury (CCl) is a model of peripheral neuropathy and involves partial sciatic nerve ligation and spinal nerve ligation. It is used to investigate both the pathophysiology and potential therapeutic agents for the treatment of neuropathic pain (4).

In experimental studies, drug therapy is usually applied immediately after $\mathrm{CCl}$. In clinical practice, however, patients receive medical treatment after the onset of neuropathic pain symptoms. In these situations, where the nerve damage is increased, treatment is more difficult. To simulate this chronic process, we performed $\mathrm{CCl}$ for 7 days, followed by curcumin, tramadol, and chronic constriction release (CCR) treatment.

The goal of the present study was to compare the efficacy of curcumin, tramadol, and CCR treatment, applied individually or together, in a rat model of sciatic nerve injury. For this purpose, we used mechanical and behavioral tests, biochemical cytokine expression analyses, and morphological examinations using electron microscopy.

\section{MATERIAL and METHODS}

Experimental procedures were approved by the local ethics committee (Protocol No. 376/2014) and conducted according to the healthcare guidelines for laboratory animals and the Universal Declaration on Animal Welfare. The animals were provided by the Center for Medical and Surgical Research. The animals were housed in separate cages, with day and night cycles, and were habituated before the experiments. Access to free standard pellet and tap water ad libitum were allowed for all animals during the experiments.

Curcumin (Curcuma longa; St. Louis, MO, USA) was purchased from the Sigma-Aldrich catalog. Restricted drugs

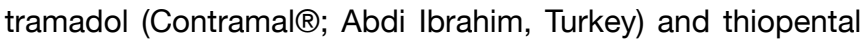
sodium (Pental Sodyum ${ }^{\circledR}$; I.E. Ulugay, Turkey) were obtained from the Department of Anesthesia with permission for the experiments.

\section{Determination of the Study Groups}

Study groups, that were determined based on the staged procedures and interventions applied to the animals, are summarized in Table I. Eighty male Sprague-Dawley rats weighing $230-250 \mathrm{~g}$ were divided into eight groups $(n=10)$. Group 1 was the sham group. Group 2 was the control group with established CCI. In Groups 3-8, CCl was also established, but experimental treatment modalities were applied in a single or combined manner during the experimental course. CCR was the only treatment performed in Group 3 (CCl-CCR). Groups $4(\mathrm{CCl}-\mathrm{C})$ and $5(\mathrm{CCl}-\mathrm{T})$ received oral curcumin and intraperitoneal tramadol, respectively. Groups 6 (CCl-CCR-C) and 7 (CCl-CCR-T) also received curcumin and tramadol after CCR, respectively. Combined curcumin and tramadol treatment was applied to Group 8 (CCl-C-T).

\section{Experimental Procedures}

The schedule of the experiment is schematically demonstrated in Table II.

\section{Surgical Procedures}

Rats were anesthetized with thiopental sodium $(40-50 \mathrm{mg} /$ $\mathrm{kg}$ ) intraperitoneally. After depilation of the right hind limb, the right sciatic nerve was exposed under aseptic conditions at the mid-thigh level beneath the gluteus and biceps femoris muscles. A 7-mm long common sciatic nerve segment proximal to the trifurcation was freed from the surrounding tissue. The procedure was ended for Group 1 (sham) at this point. In Groups 2-8, CCl was induced by placing four loose ligatures (4/0 silk suture) around the nerve at intervals of approximately $1 \mathrm{~mm}$ under an operating microscope. Then, the wound was closed. The animals were returned to their cages to recover.

On day 7 , all animals, including the sham group, were anesthetized again and their wounds were re-explored. In Groups 3, 6, and 7, CCR was performed by carefully removing the ligatures under an operating microscope. $\mathrm{CCl}$ was decompressed in this manner. The stitches were retained (Groups 2, 4, 5, and 8), and $\mathrm{CCl}$ persisted throughout the experiments.

Table I: Distribution of Study Groups according to the Procedures and Interventions

\begin{tabular}{cc}
\hline Groups $(\mathbf{n = 1 0})$ & Procedures and Interventions \\
\hline 1 (Sham) & - \\
\hline 2 (Control) & CCl \\
\hline 3 & CCl-CCR \\
\hline 4 & CCl-C \\
\hline 5 & CCl-T \\
\hline 6 & CCl-CCR-C \\
\hline 7 & CCl-CCR-T \\
\hline 8 & CCl-C-T \\
\hline
\end{tabular}

CCl: Chronic Constriction Injury, CCR: Chronic Constriction Release, C: Curcumin, T: Tramadol. 
At the end of the second stage, the animals survived for an additional 14 days. On day 21, the animals were sacrificed with high-dose thiopental sodium and exsanguination. The sciatic nerves, L4-5, and S5 (if continued with a sciatic nerve branch) dorsal root ganglia (DRGs) were dissected and removed in seven animals from each group for an enzymelinked immunosorbent assay (ELISA) assay. The remaining animals were perfused with intracardiac $2.5 \%$ glutaraldehyde in $0.1 \mathrm{M}$ phosphate buffer $(\mathrm{pH}=7.35)$ solution for histological evaluation. Nerve segments $1 \mathrm{~cm}$ long were cut proximal to the trifurcation including constricted sites, and 2-3 DRGs were collected. The freshly dissected samples were stored at $-80^{\circ} \mathrm{C}$ until analysis. The latter were fixed in $2.5 \%$ glutaraldehyde solution and sent to an associated laboratory.

\section{Drug Administrations}

Curcumin was freshly dissolved in distilled water and dimethyl sulfoxide $25 \%$ (vehicle) before administration. Tramadol was

Table II: Experimental Schedule

\begin{tabular}{ccc}
\hline Days & Procedures & Treatment \\
\hline 0 & $\mathrm{Bt}, \mathrm{CCl}$ & \\
\hline 1 & & \\
\hline 3 & $\mathrm{Bt}$ & \\
\hline
\end{tabular}

\begin{tabular}{lll}
\hline 6 & & \\
\hline 7 & Bt, CCR & DA \\
\hline & DA \\
\hline & DA \\
\hline & DA \\
\hline 13 & DA \\
\hline & & DA \\
\hline & & DA \\
\hline & & DA \\
\hline 17 & DA \\
\hline & & DA \\
\hline & DA \\
\hline & & DA \\
\hline & DA $S$ & DA \\
\hline
\end{tabular}

Bt: Behavioural tests, CCI: Chronic Constriction Injury, CCR: Chronic Constriction Release, DA: Drug administrations (Curcumin/Tramadol), S: Sacrification. diluted in distilled water (vehicle) for administration in proper volumes. Seven days after $\mathrm{CCl}$, Groups 4 and 6 started to receive oral curcumin $(100 \mathrm{mg} / \mathrm{kg}$ ) daily for 14 days. Meanwhile, intraperitoneal injections of tramadol $(10 \mathrm{mg} / \mathrm{kg})$ were started and continued daily for 14 days in Groups 5 and 7 . Both agents were administered during the same period in Group 8. The animals that were not treated with the relevant drugs received empty vehicle solutions orally or intraperitoneally.

\section{Behavioral Tests}

Behavioral tests were performed on days 0 (baseline testing), $3,7,13,17$, and 21 , between the hours of 9:00 a.m. and 4:00 p.m. by the same observer who was experienced on animal behavior. On each testing day, the rats were allowed to become accustomed to the laboratory environment. One-hour intervals between tests were provided for each animal.

Thermal Hyperalgesia Test: The withdrawal responses to a noxious heat stimulus were evaluated with a thermal hyperalgesia (TH) device (Commat Ltd., Ankara, Turkey). Rats were placed on a glass surface restricted with a Plexiglas box and acclimated for approximately 30 minutes before testing. The midplantar region of the right hind paw was exposed to constant heat generated from a radiant infrared light beam source at $20 \%$ thermal power unit of the device. Mean values of five measurements (at 5-minute intervals) were taken as withdrawal thresholds. A cut-off time of 22 seconds was maintained to prevent inadvertent tissue injury. Data were expressed as mean \pm standard deviation for withdrawal latency in seconds.

Dynamic Plantar Test: Mechanical allodynia (MA) was evaluated using a dynamic plantar aesthesiometer (Ugo Basile; Comerio-Varese, Italy). Rats were acclimated in restricted Plexiglas cages on perforated wire platforms for 30 minutes. A movable touch-stimulator unit with a von Frey-type $0.5 \mathrm{~mm}$ filament exerted increasing force under the midplantar region of the right hind paw until the animal twitched its paw. Five measurements were taken at 5-min intervals and data were expressed as mean \pm standard deviation for withdrawal latency in grams. A cut-off value of $50 \mathrm{~g}$ in 20 seconds was determined.

Cold Plate Test: Cold-induced pain behavior was quantitatively examined using a cold plate test device (Ugo Basile; ComerioVarese, Italy). The rat was placed on a metal plate kept at a cold temperature $\left(4 \pm 1^{\circ} \mathrm{C}\right)$ surrounded by a round Plexiglas chamber. Paw withdrawal latency of the right hind paw were recorded for a period of 3 minutes as a response to cold. Similar movements of other paws were excluded.

\section{TNF- $a$ and IL-10 ELISA Immunoassay}

On day 21, the nerve and DRG samples were homogenized in ice-cold phosphate-buffered saline (PBS) and centrifuged at $1500 \mathrm{~g}$ for 15 minutes. Supernatants were obtained. Total protein concentration was measured using the Bradford protein assay in the tissue supernatants. Tissue TNF- $a$ (Boster Biological Technology Co. Ltd., Pleasanton, CA, USA) and IL10 (BioSource International Inc., Camarillo, USA) levels were spectrophotometrically analyzed using a BioTek ${ }^{\mathrm{TM}}$ elx800 
Multilabel Plate Reader (BioTek Instruments Inc., Winooski, VT, USA) at $450 \mathrm{~nm}$ absorbance. Results were expressed as picograms of TNF- $a$ and IL-10 protein to $100 \mu \mathrm{gr}$ of total protein in the supernatant.

\section{Histological Analysis}

After 24 hours of fixation in $2.5 \%$ glutaraldehyde in $0.1 \mathrm{M}$ phosphate buffer, samples of the nerve segments and DRGs were rinsed and postfixed with $1 \%$ osmium tetroxide in 0.1 $\mathrm{M}$ phosphate buffer for 2 hours at room temperature. All specimens were then dehydrated in graded solutions of ethyl alcohol and embedded in epon-resin. Sections of $4 \mu \mathrm{m}$ in thickness were cut in various depths of the samples and stained with toluidine blue. The sections were blindly examined under light microscope at 40× magnification (Olympus BX5; Tokyo, Japan) by a histologist. Degenerated axons were determined based on two criteria involving myelin debris formation and finer degeneration in axons. The degenerated and normal axons were counted in 10 visual fields of each specimen and degenerated/normal axons ratios (deg/nor) were calculated.

\section{Statistical Analysis}

Data analysis was performed using the SigmaStat 3.5 package program. Descriptive statistics of continuous variables were given as mean \pm SEM. Two-way repeated measures ANOVA (One-Factor Repetition) analyses were used for data analysis. Multiple comparisons were made using the Tukey test. $p$ values of $<0.05$ were considered statistically significant.

\section{RESULTS}

$\mathrm{CCl}$ was clinically established until day 7 due to observations from behavioral tests in all representative study groups (Groups 2-8) compared with Group 1 (sham). The results of each behavioral test after day 7 , when drug administration without surgical decompression (release) was initiated, are presented below.
Thermal Hyperalgesia Test: Paw withdrawal latency decreased in Groups (2--8) following CCl compared to Group 1 (sham). After day 7, increases in withdrawal latency were steadily significant only in the $\mathrm{CCl}-\mathrm{C}-\mathrm{T}$ group on days 13 $(p<0.05), 17$, and $21(p<0.001)$ compared with those in the $\mathrm{CCl}$ group. In the CCl-CCR-T and CCl-T groups, TH latency was significantly elevated only on day $21(p<0.001)$. However, TH response did not attenuate in the $\mathrm{CCl}-\mathrm{CCR}, \mathrm{CCl}-\mathrm{C}$, and $\mathrm{CCl}-$ CCR-C groups (Figure 1).

Dynamic Plantar Test: After drug administrations were initiated without CCR intervention on day 7 , paw withdrawal threshold increased in the CCI-CCR-T group on days 13, 17 $(p<0.05)$, and $21(p<0.01)$ compared with the $\mathrm{CCl}$ group. The $\mathrm{CCl}-\mathrm{T}$ group exhibited increased paw withdrawal threshold only on day 21 compared with the $\mathrm{CCl}$ group. No significant changes were noted in paw withdrawal thresholds of the $\mathrm{CCl}$ $\mathrm{CCR}, \mathrm{CCl}-\mathrm{C}$, and $\mathrm{CCl}-\mathrm{CCR}-\mathrm{C}$ groups (Figure 2).

Cold Plate Test: There was no significant difference between the groups.

\section{TNF-a and IL-10 ELISA Immunoassay}

Sciatic Nerve: TNF-a levels were significantly higher (19866.5) $(p<0.05)$ in the $\mathrm{CCl}$ group than the other groups. The sciatic nerve TNF-a levels in the CCl-CCR-T group were significantly lower than the other groups (8333.3; $\mathrm{p}<0.05)$. IL-10 levels were significantly higher in the CCI-CCR-T group (24164.2; $\mathrm{p}>0.05)$. IL-10 levels were lower in the $\mathrm{CCl}$ and $\mathrm{CCl}-\mathrm{T}$ groups than in the other groups (156338.2, 14298.9, respectively) (Figure 3).

Dorsal Root Ganglion: TNF-a levels were significantly higher in the $\mathrm{CCl}$ group than the other groups $(52869.9 ; \mathrm{p}<0.05)$. TNF-a levels in the CCI-CCR-T group were significantly lower than those in the $\mathrm{CCl}$ group (25995.4; $\mathrm{p}<0.05)$. IL-10 levels were significantly higher in the $\mathrm{CCl}-\mathrm{T}$ group $(67397.4 ; \mathrm{p}<0.05)$; the second highest value was in the CCI-CCR-T group. In the $\mathrm{CCl}$ and $\mathrm{CCl}-\mathrm{C}-\mathrm{T}$ groups, IL-10 levels were lower than those in the other groups (24184.07 and 25861.6) (Figure 3).

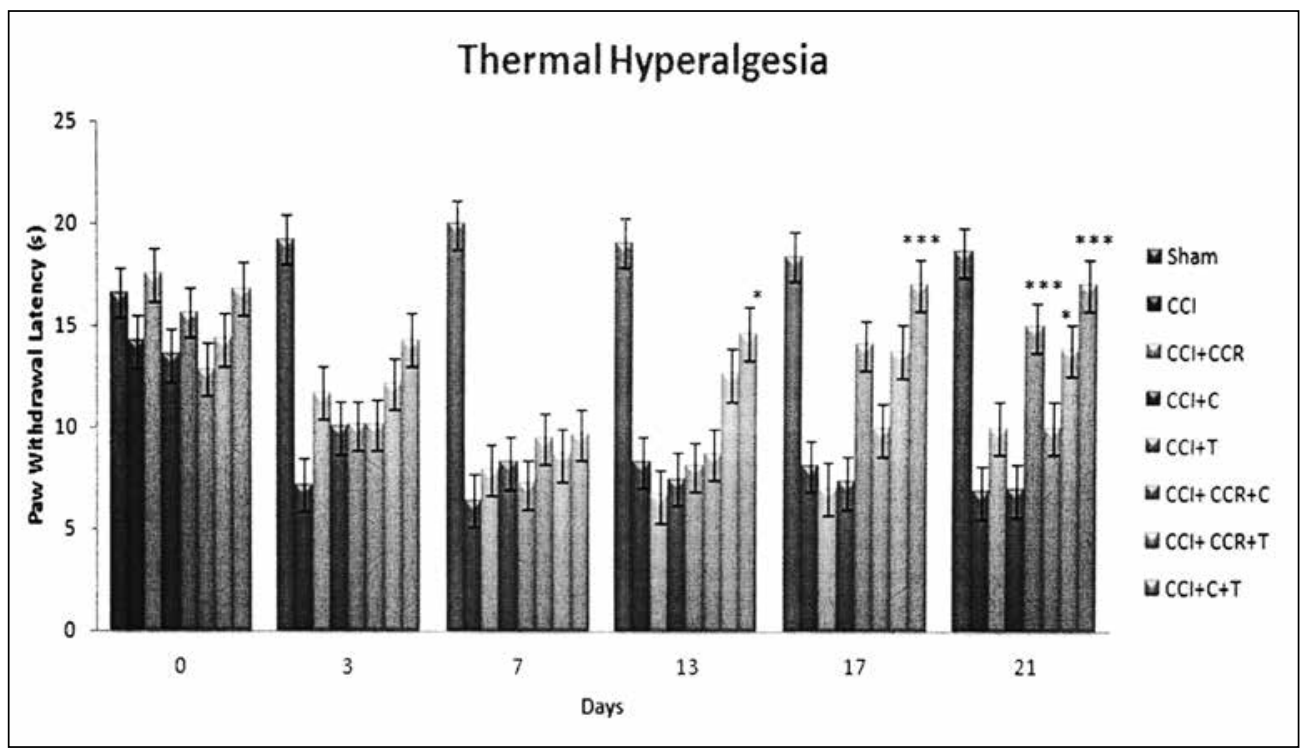

Figure 1: Effects of all treatment on the paw withdrawal thermal latency. (CCl: Chronic Constriction Injury, CCR: Chronic Constriction Release, C: Curcumin, T: Tramadol). 


\section{Results of the Histological Study}

Normal sciatic nerve with myelinated large axons was apparent on histological examination in the sham group. The $\mathrm{CCl}$ group showed evidence of $\mathrm{CCl}$ with degenerated axons and myelin sheaths. Axonal degeneration and demyelination was also evident only in the CCl-T group. Weak axonal regeneration accompanied degeneration in the $\mathrm{CCl}-\mathrm{C}$ and $\mathrm{CCl}-\mathrm{C}-\mathrm{T}$ groups without CCR intervention. In the CCl-CCR group, remarkable features of axonal regeneration were evident. The numbers of regenerated axons increased in the CCI-CCR-C and CClCCR-T groups, which were more evident in the CCl-CCR-C group (Figure $4 \mathrm{~A}-\mathrm{H}$ ).

In the present study, a significant decrease was found in the deg/nor axons ratios of Groups 3, 4, 6, 7, and 8 compared with that of Group $2(p<0.05)$ (Figure 5).

Major pathological changes associated with $\mathrm{CCl}$ in DRGs included vacuolization, increase in the sizes of cells due to

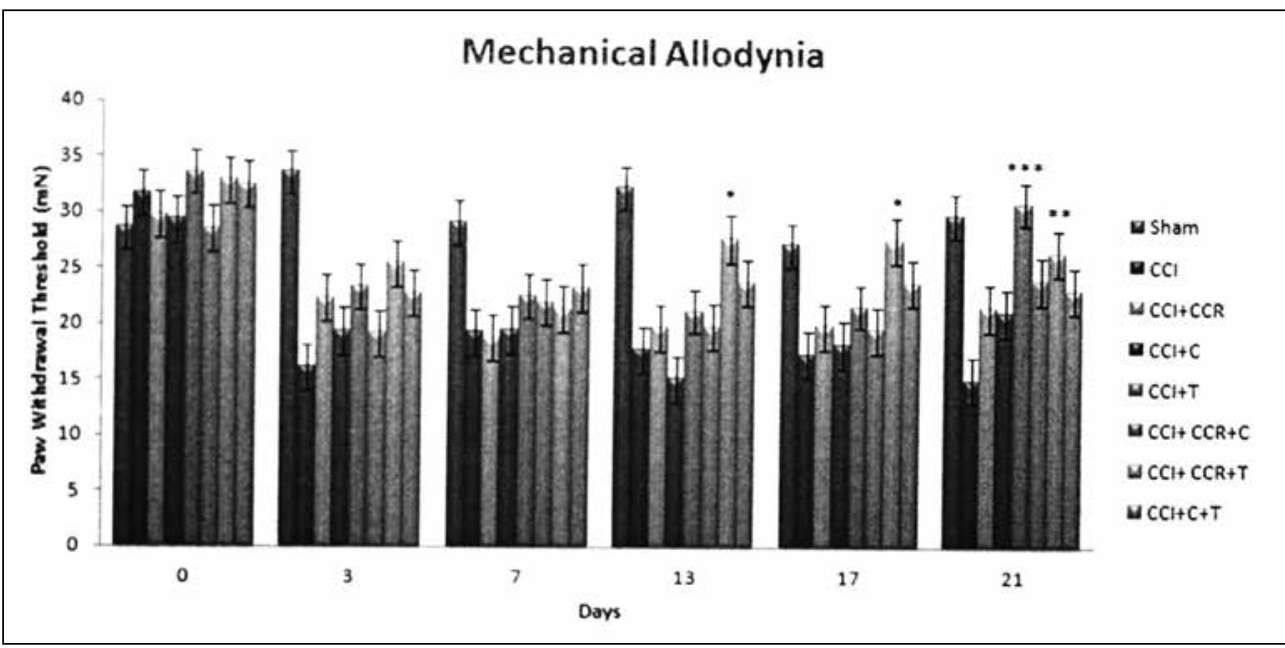

Figure 2: Effects of all treatment on the paw withdrawal mechanical threshold.

(CCl: Chronic Constriction Injury, CCR: Chronic Constriction Release, C: Curcumin T: Tramadol).

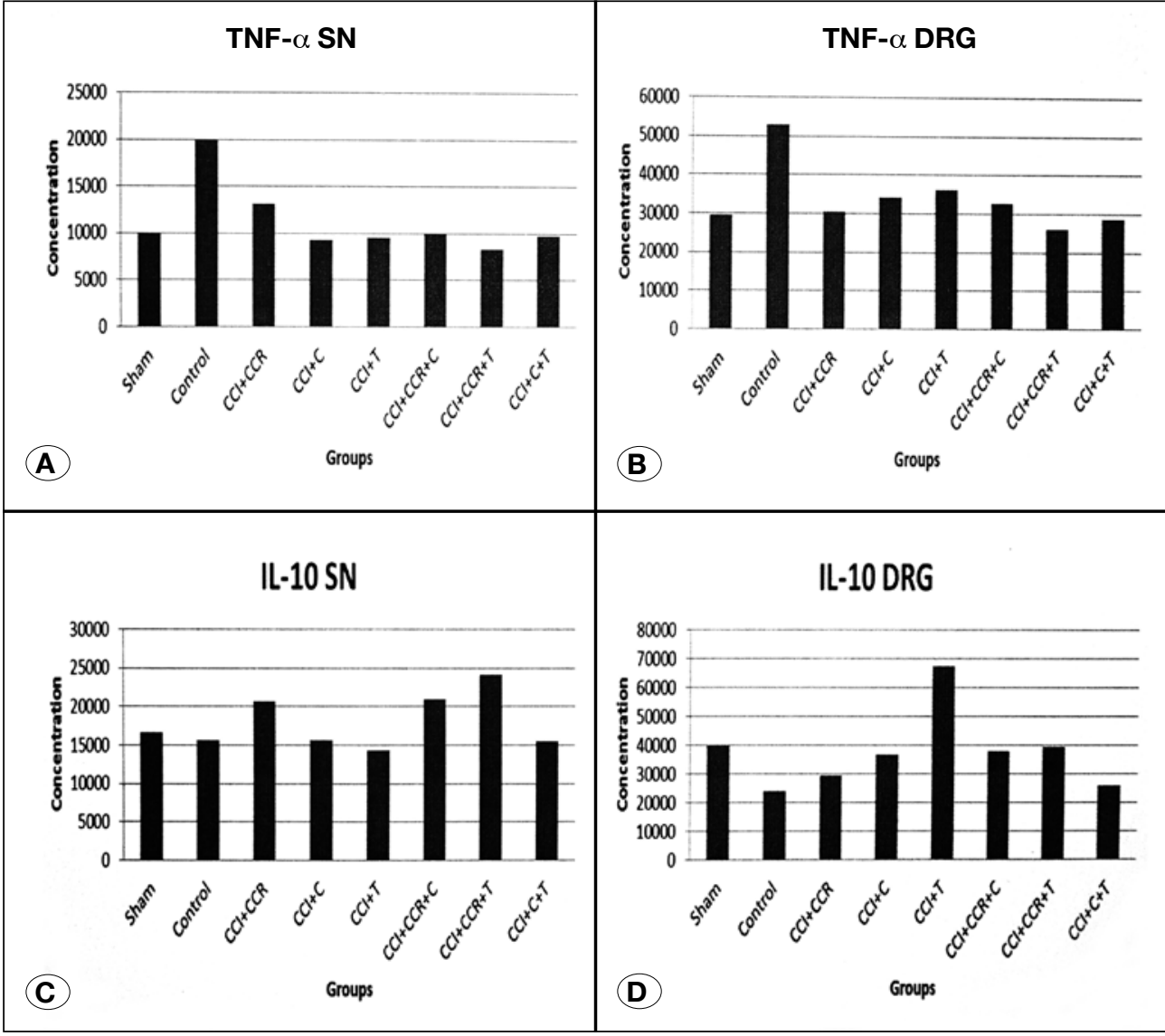

Figure 3: TNF- $a$ and IL-10 levels in sciatic nerve (SN) and DRG in all groups. A) TNF-a level in sciatic nerve. B) TNF-a level in DRG. C) IL-10 level in sciatic nerve. D) IL-10 level in DRG. (CCl: Chronic Constriction Injury, CCR: Chronic Constriction Release, C: Curcumin, T: Tramadol). 


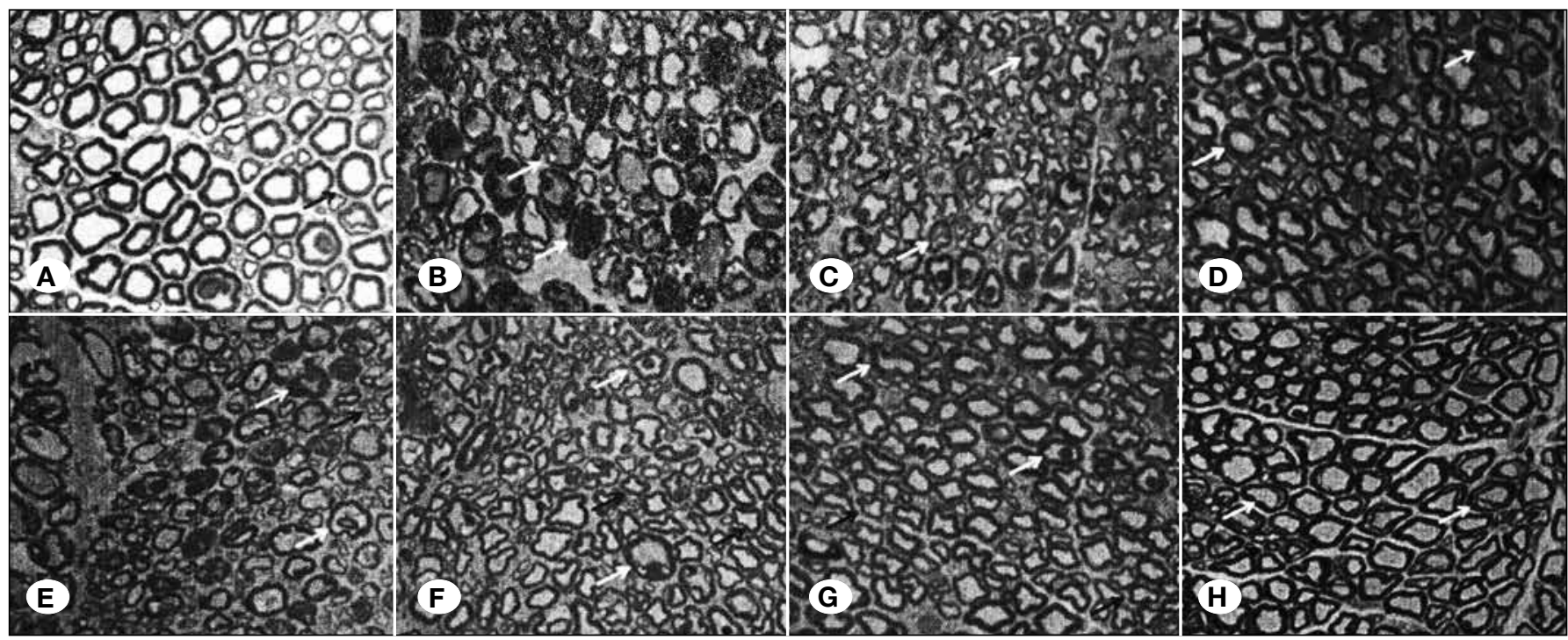

Figure 4: Cross sections of the affected regions of the sciatic nerves stained with toluidine blue at 40X magnification. A) Group 1(Sham). Healthy neural structure with large axons and thick myelin. B) Group 2 (CCl) remarkable degeneration of axons and myelin sheaths. C) Group 3 (CCl-CCR). Some extent of regeneration was seen following CCR. D) Group 4 (CCl-C) weak axonal regeneration accompanied the degeneration. E) Group 5 (CCl-T) degenerative features of the axons and myelin sheaths similar to Group 2. F) Group 6 (CCl-CCR-C) and G) Group 7 (CCl-CCR-T) Drug administration (Curcumin or Tramadol) following CCR accelerated regeneration of axons. H) Group 8 (CCl-C-T) weak axonal regeneration also accompanied degeneration as shown in group 4. (Yellow arrow indicates degenerated axons; black arrow indicates normal or regenerated axons).

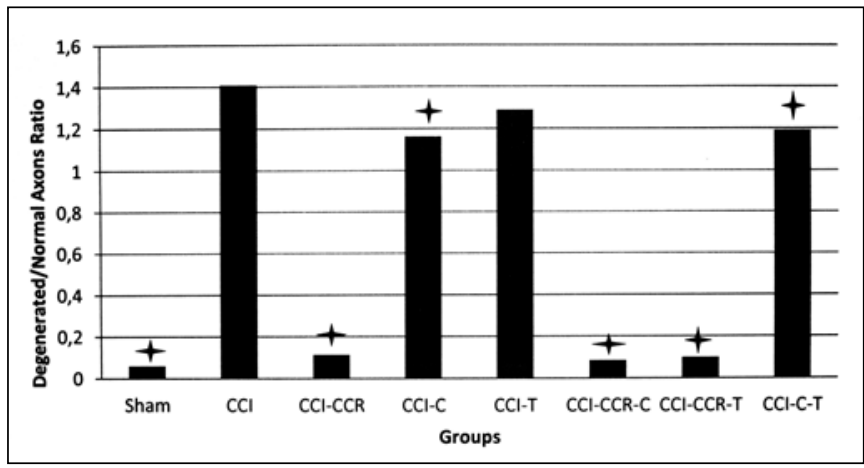

Figure 5: Graphic presentation of the mean degenerated/normal axon ratios ( ${ }^{*} p<0.05$ compared to Group 2 ).

swelling, and loss of membrane cell lines and nuclei (Figure 6). In CCR-performed groups (Groups 3, 6, and 7), these changes decreased but persisted. However, DRGs of the animals in the sham group showed normal morphology with regularly arranged structures.

\section{DISCUSSION}

Our study findings indicate that neuropathic pain prevention in the CCl-CCR-T group was more effective than that in the $\mathrm{CCl}-\mathrm{C}$ group, as shown by the decreased TNF-a and increased IL-10 levels in the sciatic nerves. On the other hand, histological examination showed that curcumin treatment was associated with the regeneration of degenerated nerve tissues compared with the other treatments.
The present study demonstrates that $\mathrm{CCl}-\mathrm{C}$ and $\mathrm{CCl}-\mathrm{CCR}$ treatments were not significantly effective in reducing $M A$ and TH. MA and TH were significantly attenuated in all tramadoltreated groups (CCl-T, CCl-CCR-T, and CCl-C-T). With $\mathrm{CCl}$ CCR-T treatment, TNF-a levels were significantly low in the sciatic nerve tissue and DRG, and IL-10 levels were significantly high in the sciatic nerve tissue. IL-10 levels were higher in DRGs of CCl-T and CCl-CCR-T groups. Our histological examinations demonstrated that drug administration following CCR accelerated regeneration of axons, but curcumin by itself led to higher axonal regeneration than tramadol only in the sciatic nerve tissue. CCR alone was more effective in the formation of axonal regeneration than curcumin or tramadol alone.

In a previous study, rats administered curcumin for 7 and 14 days showed improvement in the symptoms of allodynia and hyperalgesia (9). Another study showed that curcumin administration can alleviate hyperalgesia induced by neuropathic pain (12). In our study, treatment with curcumin with or without CCR did not inhibit hyperalgesia, but curcumin in combination with tramadol was effective in preventing hyperalgesia. Sakakiyama et al.(13) suggested that repeated tramadol administration is an effective treatment for neuropathic pain in rats. CCR alone did not act on hyperalgesia. Similar to our results, studies have shown that tramadol was more effective in treatment of neuropathic pain with the use of different drugs available $(8,19)$.

Some pro-inflammatory cytokines, such as TNF-a, play an important role in the development of hyperalgesia. Our results are in accordance with these findings; both TNF-a and $\mathrm{MH}$ 


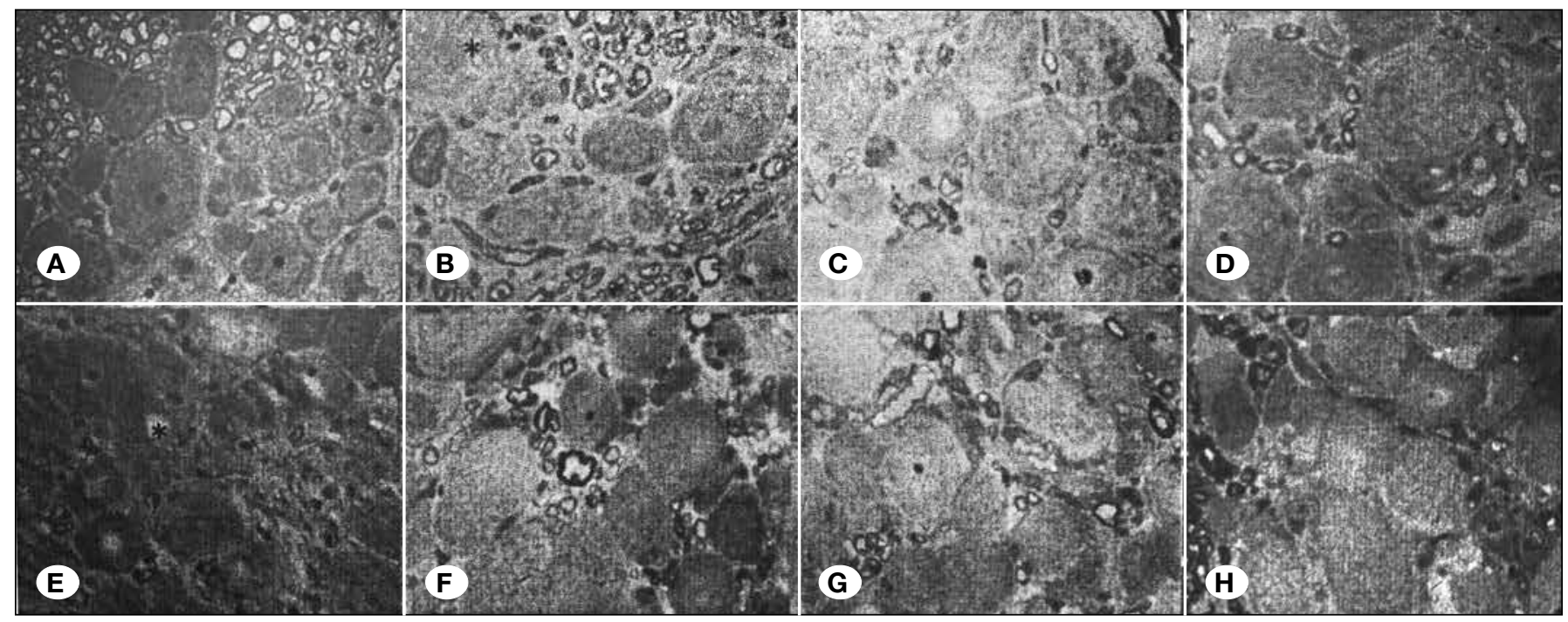

Figure 6: Cross sections of the associated DRGs of the sciatic nerves according to groups. A) Group 1(Sham), B) Group 2 (CCI), C) Group 3 (CCl-CCR), D) Group 4 (CCl-C), E) Group 5 (CCl-T), F) Group 6 (CCl-CCR-C), G) Group 7 (CCl-CCR-T), H) Group 8 (CCl$\mathrm{C}-\mathrm{T})$. * indicated vacuolisation (toluidine blue at 40X magnification).

were increased in the control group. Curcumin has been shown to inhibit these cytokines in a previous study (17). We found that $\mathrm{CCl}-\mathrm{C}$ treatment was more effective than $\mathrm{CCl}-\mathrm{T}$ in reducing TNF-a levels in nerve tissues. However, unlike the literature, curcumin could not sufficiently prevent hyperalgesia. On the other hand, CCI-CCR-T treatment was found to be more effective than $\mathrm{CCl}-\mathrm{C}$ in the nerve tissue. $\mathrm{CCl}-\mathrm{CCR}$, that is surgical treatment, was not enough to decrease TNF-a levels in the nerve tissue. When evaluated with DRG, TNF-a levels were the lowest in the CCI-CCR-T group.

IL-10, a potent anti-inflammatory cytokine, is known to play a role in neuropathic pain by decreasing the synthesis of proinflammatory cytokines (1). Increased IL-10 levels decrease TNF-a levels, reducing hyperalgesia. Similar results were obtained in the present study; TNF-a levels were significantly low, whereas IL-10 was high in the sciatic nerve tissue in the $\mathrm{CCl}-\mathrm{CCR}-\mathrm{T}$ group. This inverse relationship between the two cytokines was also observed in the other groups, but was not as significant as that in the CCI-CCR-T group. However, our study could not demonstrate the relationship between these cytokines and hyperalgesia. The CCl-T group has the highest level of IL-10 in DRG, followed by the CCI-CCR-T group.

The neuroprotective effect of curcumin has been shown in different conditions, such as spinal cord injury (20), in a neurotoxic model (16). Al Moundhri et al.(2) reported that curcumin had neuroprotective effects on oxaliplatin and cisplatin neurotoxicity in rats. In that study, the curcumintreated group showed normal-looking myelinated nerve fibers with no evidence of demyelination. The cisplatin+curcumintreated group showed a marked decrease in demyelination. In our study, a remarkable degeneration of axons and myelin sheaths was observed after compression of the sciatic nerve tissue. Some extent of regeneration was seen following CCR. While curcumin without surgical constriction release $(\mathrm{CCl}-\mathrm{C})$ slightly helped regeneration, tramadol without surgical constriction release $(\mathrm{CCl}-\mathrm{T})$ did not cause the degeneration of improvement. CCR in combination with tramadol $(\mathrm{CCl}-$ CCR-T) reduced degeneration. Similar and better effects were seen with CCR in combination with curcumin (CCl-CCR-C).

In the treatment of chronic neuropathic pain induced by chronic constriction, 1) surgical constriction release only was not effective, 2) $\mathrm{CCl}-\mathrm{CCR}-\mathrm{T}$ treatment was highly effective in the symptomatic treatment of neuropathic pain, 3) curcumin was not as effective as CCl-CCR-T treatment, and. 4) CCR-C is associated with a decrease in degeneration and increase in regeneration of nerve tissue.

\section{- CONCLUSION}

Long-term use of curcumin following surgical constriction release may have beneficial effects on chronic neuropathic pain induced by chronic constriction.

\section{ACKNOWLEDGEMENT}

This paper was supported from the research council of Osmangazi University Medical Faculty Research Fund with grant number: 2014-345.

\section{REFERENCES}

1. Abarikwu SO, Durojaiye M, Alabi A, Asonye B, Akiri O: Curcumin protects against gallic acid-induced oxidative stress, suppression of glutathione antioxidant defenses, hepatic and renal damage in rats. Ren Fail 38: 321-329, 2015

2. Al Moundhri MS, Al-Salam S, Al Mahrouqee A, Beegam S, Ali BH: The effect of curcumin on oxaliplatin and cisplatin neurotoxicity in rats: Some behavioral, biochemical and histopathological studies. J Med Toxicol 9: 25-33, 2013

3. Babu A, Prasanth KG: Effect of curcumin in mice model of vincristine-induced neuropathy. Pharmaceutical Biology 53: 838-848, 2015 
4. Berrocoso E, De Benito MD, Mico JA: Role of serotonin 5-HT1A and opioid receptors in the antiallodynic effect of tramadol in the chronic constriction injury model of neuropathic pain in rats. Psychopharmacology (Berl) 193: 97-105, 2007

5. Clark AK, Old EA, Malcangio M: Neuropathic pain and cytokines: Current perspectives. J Pain Res 21:803-814; 2013

6. George A, Buehl A, Sommer C: Tumor necrosis factor receptor 1 and 2 proteins are differentially regulated during Wallerian degeneration of Mouse sciatic nerve. Exp Neurol 192:163166, 2005

7. Ji FT, Liang JJ, Liu L, Cao MH, Li F: Curcumin exerts antinociceptive effects by inhibiting the activation of astrocytes in spinal dorsal horn and the intracellular extracellular signalregulated kinase signaling pathway in rat model of chronic constriction injury. Chin Med J (Engl) 126:1125-1131, 2013

8. Jung KH, Lee JH, Park JW, Moon SH, Cho YS, Choe YS, Lee $\mathrm{KH}$ : Effects of curcumin on cancer cell mitochondrial function and potential monitoring with 18F-FDG uptake. Oncol Rep 35: 861-868, 2016

9. Kaneko K, Umehara M, Homan T, Okamoto K, Oka M, Oyama $\mathrm{T}$ : The analgesic effect of tramadol in animal models of neuropathic pain and fibromyalgia. Neurosci Lett 562:28-33, 2014

10. Khan J, Ramadan K, Korczeniewska O, Anwer MM, Benoliel $\mathrm{R}$, Eliav E: Interleukin-10 level in rat models of nerve damage and neuropathic pain. Neurosci Lett 10: 592-599, 2015

11. Kim SH, Chung JM: An experimental model for peripheral neuropathy produced by segmental spinal nerve ligation in the rat. Pain 3: 355-363, 1992

12. Maithilikarpagaselvi N, Sridhar MG, Swaminathan RP, Zachariah P: Curcumin prevents inflammatory response, oxidative stress and insulin resistance in high fructose fed male Wistar rats: Potential role of serine kinases. Chem Biol Interact 25: 187-194, 2016
13. Mei XP, Chen L, Wang W, Wu D, Wang LY, Zhang T, Zhang $\mathrm{H}, \mathrm{Xu}$ LX, Li YQ: Combination of tramadol with minocycline exerted synergistic effects on a rat model of nerve injuryinduced neuropathic pain. Neurosignals 21:184-196, 2013

14. Sahbaie P, Sun Y, Liang DY, Shi XY, Clark JD: Curcumin treatment attenuates pain and enhances functional recovery after incision. Anesth Analg 118: 1336-1344, 2014

15. Sakakiyama M, Maeda S, Isami K, Asakura K, So K, Shirakawa $\mathrm{H}$, Nakagawa T, Kaneko S: Preventive and alleviative effect of tramadol on neuropathic pain in rats: Roles of $a_{2}$ adrenoceptors and spinal astrocytes. J Pharmacol Sci 124: 244-257, 2014

16. Sharma S, Chopra K, Kulkarni SK: Effect of insulin and its combination with resveratrol or curcumin in attenuation of diabetic neuropathic pain: Participation of nitric oxide and TNF-alpha. Phytother Res 21: 278-283, 2007

17. Singh AK, Vinayak M: Curcumin attenuates CFA induced thermal hyperalgesia by modulation of antioxidant enzymes and down regulation of TNF-a, IL-1 $\beta$ and IL-6. Neurochem Res 40: 463-472, 2015

18. Tang DT, Barbour JR, Davidge K, Yee A, Mackinnon SE: Nerve entrapment: Update. Plast Reconstr Surg 135:199e-215e, 2015

19. Zhang J, Wu D, Xie C, Wang H, Wang W, Zhang H, Liu R, Xu LX, Mei XP: Tramadol and propentofylline coadministration exerted synergistic effects on rat spinal nerve ligation-induced neuropathic pain. PLoS One 29:e72943, 2013

20. Zhao X, Xu Y, Zhao Q, Chen CR, Liu AM, Huang ZL: Curcumin exerts antinociceptive effects in a mouse model of neuropathic pain: Descending monoamine system and opioid receptors are differentially involved. Neuropharmacology 62: 843-854, 2012 